\title{
Tip-Enhanced Raman Spectroscopy: From Concepts to Practical Applications
}

\author{
Nan Jiang ${ }^{\text {a, }}$, Dmitry Kurouski ${ }^{\text {b }}$, Eric A. Pozzi ${ }^{\text {b }}$, Naihao Chiang ${ }^{\text {c }}$, Mark C. Hersam ${ }^{\text {b, c, d }}$, and \\ Richard P. Van Duyne ${ }^{\text {b, c, * }}$ \\ ${ }^{a}$ Department of Chemistry, University of Illinois at Chicago, Illinois 60607, United States \\ ${ }^{b}$ Department of Chemistry, ${ }^{\mathrm{c}}$ Applied Physics Graduate Program and ${ }^{\mathrm{d}}$ Department of Materials Science and \\ Engineering, Northwestern University, Evanston, Illinois 60208, United States \\ *Corresponding authors: N.J. (email: njiang@uic.edu), R.P.V.D. (email: vanduyne@ northwestern.edu)
}

\begin{abstract}
:
Tip-enhanced Raman spectroscopy (TERS) is a powerful technique that integrates the vibrational fingerprinting of Raman spectroscopy and the sub-nanometer resolution of scanning probe microscopy (SPM). As a result, TERS is capable of obtaining chemical maps of analyzed specimens with exceptional lateral resolution. This is extremely valuable for the study of interactions between molecules and substrates, in addition to structural characterization of biological objects, such as viruses and amyloid fibrils, 2D polymeric materials, and monitoring electrochemical and photo-catalytic processes. In this mini-review, we discuss the most significant advances of TERS, including: super high resolution chemical imaging, monitoring of catalytic processes, incorporation of pulsed-excitation techniques, single-site electrochemistry, biosensing, and art conservation. We begin with a short overview of TERS, comparing it with other surface analytical techniques, followed by an overview of recent developments and future applications in TERS.
\end{abstract}




\section{Introduction}

The ability to observe chemical process on solid surfaces at molecular length scales makes manipulating and controlling reactions at the single molecular level possible. Probing individual molecules and surface sites allows one to obtain information that may otherwise be obscured due to ensemble averaging, making nanoscale spatial resolution important. Among the various classes of techniques utilized to probe solid surfaces, surface vibrational spectroscopy has gained enormous attention due to its ability to understand the structure-property relationship of nanoscale structures and their chemical interactions with solid surfaces. However, classical spectroscopic techniques, including infrared, ultraviolet-visible, Raman, and fluorescence spectroscopy, which are commonly used for such studies, are limited in terms of their spatial resolution to about half the wavelength of the light because of the Abbe diffraction limit. Recently invented super-resolution microscopy, such as photoactivated localization microscopy (PALM) and direct stochastical optical reconstruction microscopy (d-STORM), have provided a pathway for overcoming the Abbe diffraction limit in selected cases.[1-3], However, superresolution microscopy has a major drawback, namely it requires labeling since not every molecule is fluorescent.

At the same time, Raman and IR spectroscopy are indispensable tools for the collection of chemical signatures pertaining to surface defects, mechanical deformation and thermal properties. Infrared spectroscopy and Raman spectroscopy have been extensively used for probing the vibrational states of gases, liquids and solids. In infrared spectroscopy, molecules absorb incident photons having energies equal to the energy differences between the ground and vibrational excited state of each IR-active molecular normal mode. On the other hand, in Raman spectroscopy, a molecule is first excited to a virtual or real electronic state and then relaxes back to the ground state concomitant with the emission of a photon with an energy different from the incident wavelength by an amount equal to a vibrational quantum (inelastic scattering). Both techniques produce weak signals and generally require a large number of molecules to produce detectable signals.

Scanning probe microscopy (SPM) does not require light for sample imaging. Instead, a metal or silicon probe is used to obtain topographic information and physical properties of analyzed specimens. There are two major modalities of SPM: scanning tunneling microscopy (STM) and atomic force microscopy (AFM). In addition to the topography, SPM can be used to acquire electronic signatures of nanostructures. For instance, STM inelastic electron tunneling spectroscopy (IETS) has made it possible to identify the chemical fingerprint of a molecule adsorbed on a conducting surface.[4] This technique, however, requires the sample to be measured at cryogenic temperatures to avoid thermal smearing of electron energies. The IETS spectra have poorly understood selection rules, and the number of observable modes is limited compared to other vibrational spectroscopic techniques.

In 2014, high-quality tip-enhanced IR spectroscopy was reported by Lu et al.[5] Enhanced signal was collected from samples as small as monolayer islands, and the authors 

microscopy (s-NSOM) systems. An estimated 300 molecules interacting with the AFM tip corresponded with an experimental sensitivity of $\sim 30$ molecules. However, optimizing the tip plasmon resonance to enhance the entire broadband spectrum required for IR spectroscopy needs to be further developed.

Tip-enhanced Raman spectroscopy (TERS) is a new powerful analytical technique that is capable of unraveling structural organization of the analyzed specimens with sub-nanometer spatial resolution. In TERS, scanning probe microscopy is coupled with Raman spectroscopy. Illumination of noble metal nanostructures at the apex of a scanning probe with electromagnetic radiation causes collective electron oscillations on the metal surface, known as localized surface plasmon resonances (LSPR). LSPR induces intense local electromagnetic fields, causing a drastic $\left(10^{8}\right)$ enhancement of the Raman scattering signals emanating from molecules located in a close vicinity to the metal surface. Therefore, spatial resolution in TERS is determined by the confinement of the electromagnetic field and not by the wavelength of the illuminating light [69]. In addition to continuous-wave excitation, pulsed electromagnetic radiation can be used. This allows for studying molecular dynamics near plasmonic surfaces, which elucidates mechanisms of plasmon-mediated chemical reactions, such as plasmonically-enhanced photocatalytic or photovoltaic processes.

Optical excitation of a noble metal SPM probe creates a highly confined electromagnetic field at the tip-sample junction, which provides significant enhancement to the Raman scattering, infrared absorption, and fluorescence signals. With the presence of a plasmonic metal tip in close proximity to the surface (usually within a few nanometers), field enhancement allows vibrational signatures of one or a few molecules to be quantitatively detected and unambiguously identified. Unlike fluorescence spectroscopy, Raman spectroscopy is a relatively non-perturbative technique that does not require labels. The use of excitation overlapping the molecular resonance of the analyte can provide a resonance Raman contribution and a subsequent enhancement on the order of $10^{4}-10^{7}$. With this contribution, single-molecule detection can be achieved. Single-molecule detection was confirmed using a low surface coverage argument, but also included spectral fluctuations and discrete signal losses as further confirmation [10]. The first single-molecule work combined with STM topographic scanning was published by the Pettinger group, in which they analyzed BCB molecules on a smooth Au substrate using an Ag tip.[11] Sonntag et al. illustrated single-molecule detection using molecular diffusion and frequency shifts created by using the isotopically labeled R6G, so called the isotopologue approach, originally developed for single-molecule surface-enhanced Raman spectroscopy (SMSERS) [12]. The TERS signal switches between the isotopes as the molecules diffuse in and out the tip-sample junction. The detection of vibrations from single molecules makes TERS a powerful tool to study site-specific chemical structure and reactivity.

\section{Concepts}

\subsection{Spatial Resolution.}


The sub-nanometer spatial resolution of TERS allows for the unraveling of moleculesubstrate interactions. Moreover, using TERS, individual molecules can be imaged on atomically flat metal surfaces. In the first TERS paper, Stöckle et al. recorded 25 spectra along the boundary of (BCB) film dispersed on a glass support using a Ag-coated AFM tip [6]. It was shown that only Raman spectra acquired over the BCB region exhibited the signature of the dye molecule. Stöckle et al. concluded that spatial resolution is limited to a size of an apex of the metal-coated tip.

Concurrent SPM imaging supplements TERS with topographic information. More importantly, monitoring spectral changes when the tip is moved across the surface can address the spatial resolution directly. Both Deckert[13] and co-workers and Kawata[14] and co-workers have independently demonstrated sub-2 $\mathrm{nm}$ resolution TER images under ambient conditions (Figure 1 a, b). Published in Nature in 2013, Zhang et al. has further energized this field with their demonstration of sub-nm spatial resolution in the detected Raman signal of a porphyrin under ultrahigh vacuum conditions, as shown in Figure 1c.[15] The obtained TER images allow for visualization of the inner structure of a single molecule. Also demonstrated in this work was the ability to correlate the observed Raman signal with a molecule's local environment using TER imaging. This work has raised single molecule chemical imaging to an unprecedented level and sets the stage for future super high-resolution chemical imaging studies.

\section{a. Ambient - Single Amyloid Fibril}

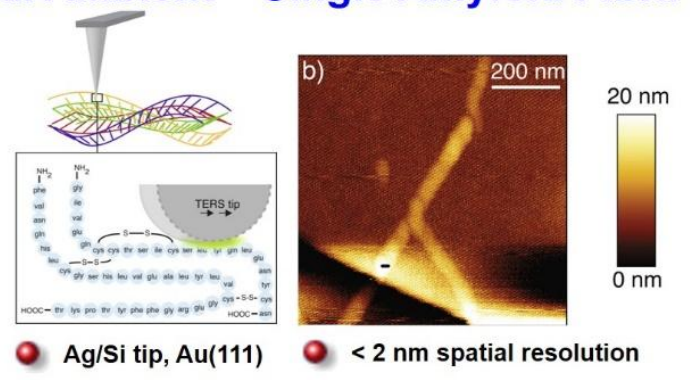

b. Ambient - Single Carbon Nanotube
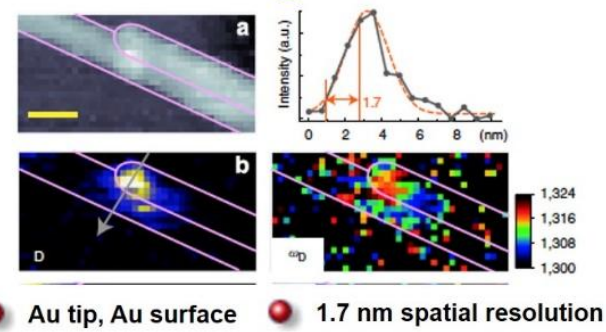

c. UHV $-\mathrm{H}_{2}$ TBPP
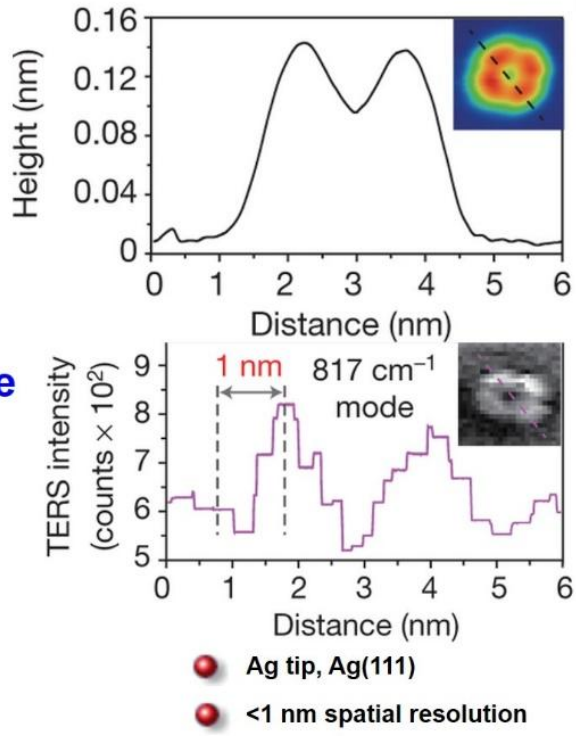

Figure 1. (a) $<2 \mathrm{~nm}$ spatial resolution TERS of single amyloid fibril. (b) $1.7 \mathrm{~nm}$ spatial resolution TERS of single carbon nanotubes. (c) <1nm spatial resolution UHV-TERS of single H2TBPP molecule. Reprinted with permission from ref $12,13,14$. Copyright 2012 Wiley, 20132014 Macmillan Publishers.

\subsection{Chemical Information}


Another advantage of optical spectroscopy is its abundant chemical information. Chemical identification considerably enhances the usefulness of SPM, especially on poorly defined surfaces of technological interest. In 2012, Jiang et al., for the first time, resolved multiple vibrational modes for large polyatomic molecular adsorbates on a solid surface in collected TER spectra obtained concurrently with molecular resolution topographic SPM imaging.[16] Furthermore, electronic structure calculations and simulated Raman spectra were carried out using density-functional theory. The atomic motions involved in each mode have been detailed. This work allows the relationship between large polyatomic molecular adsorbates and solid surfaces to be quantitatively and unambiguously interrogated. Additionally, the theories of resonance Raman scattering have predicted dependence of the Raman intensity on the excitation wavelength. Recently, multiple excitation wavelength TERS has been realized for the first time on a porphyrin molecule under UHV conditions.[17] As shown in Fig. 2, the excitation wavelengths were chosen to coincide with individual Q-bands of the porphyrin, allowing the authors to successfully resolve the spectral differences of different vibronic states. Furthermore, simultaneous collections of TERS and tip-enhanced fluorescence with the same tip were demonstrated for the first time. 

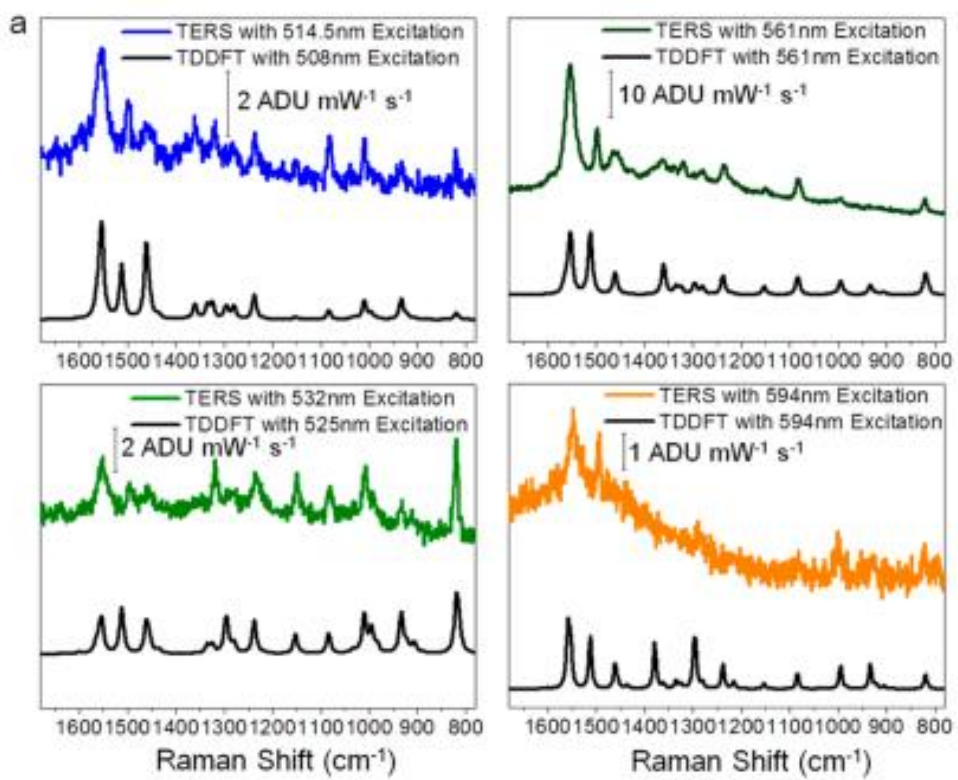

b
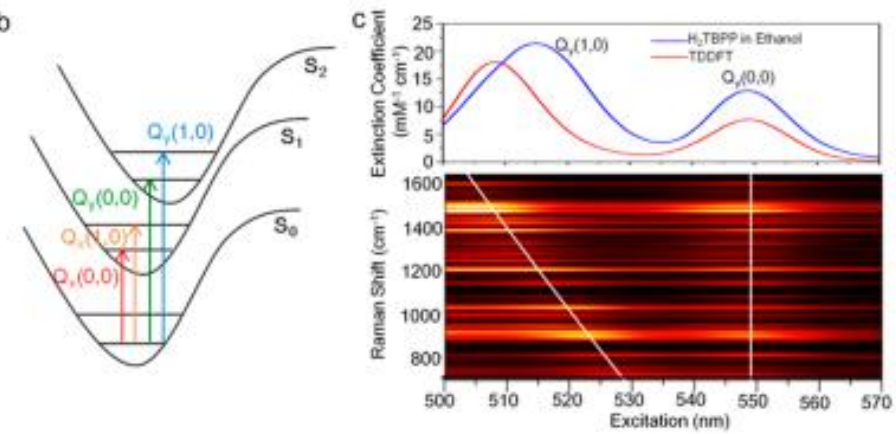

Figure 2. (a) UHV-TERS spectra of H2TBPP adlayer on $\mathrm{Ag}(111)$ (tip-engaged spectra with tip retracted spectra subtracted) with 514.5, 532, 561, and $594 \mathrm{~nm}$ excitation, and the corresponding TDDFT simulations of plasmonically enhanced Raman spectra with polarization of the excitation parallel to the tip axis. (b) Schematics of the resonance Raman process of H2TBPP. (c) TDDFT simulated excitation profile for resonance Raman spectra of H2TBPP with excitation from 490-570 nm. The white lines are the corresponding $\mathrm{Qy}(1,0)$ and $\mathrm{Qy}(0,0)$ excitation energies for each vibrational mode. Reprinted with permission from ref 16. Copyright 2014, American Chemical Society.

Beyond fingerprinting identification, TERS can also unravel the intricacies of adsorbate-substrate interactions that are inaccessible by other means. Klingsporn et al. compared the TER spectra of Rhodamine 6G (R6G) on $\mathrm{Ag}(111)$ surface at different temperatures.[18] Theoretical analysis determined that the observed spectral changes upon sample cooling were due to the strong interaction between the ethylamine substituents and the Ag surface. Liu et al. used fishing-mode TERS to observe whether the tip-surface junction is bridged by a single molecule. The clear correlations between the Raman spectra and the measured conductance indicated that TERS could be used to reveal the molecular structure in the tip-sample gap. [19] The Weckhuysen group successfully monitored a photocatalytic reaction using TERS by a dualwavelength approach.[20] A photocatalytic reduction process was induced at the apex of the tip with green laser light, while red laser light was used to monitor the transformation process during the reaction. TERS is unique in enabling the study of molecular dynamics and chemical reactions at the nanometer scale because of its localized enhancement to collect chemical signatures from 
single molecules. These results present new opportunities for the study of surface functionalization, molecular interactions, catalysis, and related interfacial phenomena.

\subsection{Temporal Resolution.}

Furthermore, optical spectroscopy includes the possibility of using ultrafast pulsed-laser excitation for temporally resolved study. Recently, improving the temporal resolution possible with TERS has proven to be non-trivial. With the predominant use of continuous-wave lasers in TERS, resolving molecular dynamics on the timescales on which they occur is seldom possible. Notable exceptions include when the process under study takes place on the timescale of seconds,[20] such that the kinetics can be monitored in successive spectral acquisitions. As a result, conventional TERS is blind to processes occurring on the order of milliseconds or faster, which unfortunately excludes the study of important transient physical phenomena.

Processes occurring at ultrafast timescales are critical to the function of chemical systems, both natural and man-made. Reaction intermediates and transition states are vital to the progression of chemical reactions and the turnover of catalysts but only exist ephemerally. Exciton generation and charge transfer dictate the performance of molecular electronics and photovoltaics, but exciton lifetimes are also short-lived. In order to spectroscopically investigate such transient processes and species, nonlinear techniques are required. Extending these studies to the nanoscale may become possible upon incorporating ultrafast spectroscopic techniques with TERS.

Raman spectroscopy is particularly well suited to study processes in chemical systems because Raman bands are specific to molecular structure and symmetry. In turn, ionization, isomerization and chemical reaction products, to name a few, are distinguishable from the initial species through their Raman vibrational signatures. Though traditional Raman spectroscopy suffers from the poor temporal resolution mentioned above, multiple pump-probe Raman techniques have been pioneered over the last few decades through the use of pulsed laser systems. These techniques notably include femtosecond-stimulated Raman spectroscopy (FSRS)[21] and coherent anti-Stokes Raman spectroscopy (CARS).[22]

Using stimulated Raman techniques, the time evolution of excited states has been discerned for numerous systems. Kukura et al. used FSRS to track the geometry of rhodopsin during the isomerization responsible for the detection of light in our retinae.[23] Fang and coworkers utilized FSRS to track the reaction coordinate of green fluorescent protein during the excited state proton transfer that leads to its fluorescence.[24] By analyzing the time evolution of chromophoric Raman band frequencies, the skeletal motions responsible for the creation of the highly fluorescent state were identified. The Wasielewski group applied FSRS to a donoracceptor molecule and were able to glean insight into the lifetime and character of the chargetransfer excited state.[25] These reports and many others highlight the capabilities of nonlinear Raman experiments toward investigating chemistry on femtosecond and picosecond timescales.

While nonlinear Raman studies are typically limited to strongly scattering analytes due to the inherent weakness of the Raman process, groups have recently begun utilizing plasmonic enhancement to extend the detection limit. The Van Duyne group pioneered surface-enhanced 
FSRS (SE-FSRS),[26] demonstrating $\sim 10^{4}$ to $10^{6}$ signal enhancement using Au nanoantennas. Further work illustrated that Fano-like couplings between molecule and plasmon cause dispersive lineshapes in SE-FSRS.[27] Surprisingly, the nearby plasmon was not found to affect the vibrational dephasing time. The Apkarian group investigated vibrational wave packet motion in surface-enhanced CARS (SE-CARS).[28] Phase fluctuations were detected over the course of several picoseconds, which the authors attributed to single-molecule detection. Researchers in the Halas group systematically verified single-molecule sensitivity in SE-CARS using the bianalyte approach, reporting a total enhancement $\sim 11$ orders of magnitude over spontaneous Raman scattering.[29]
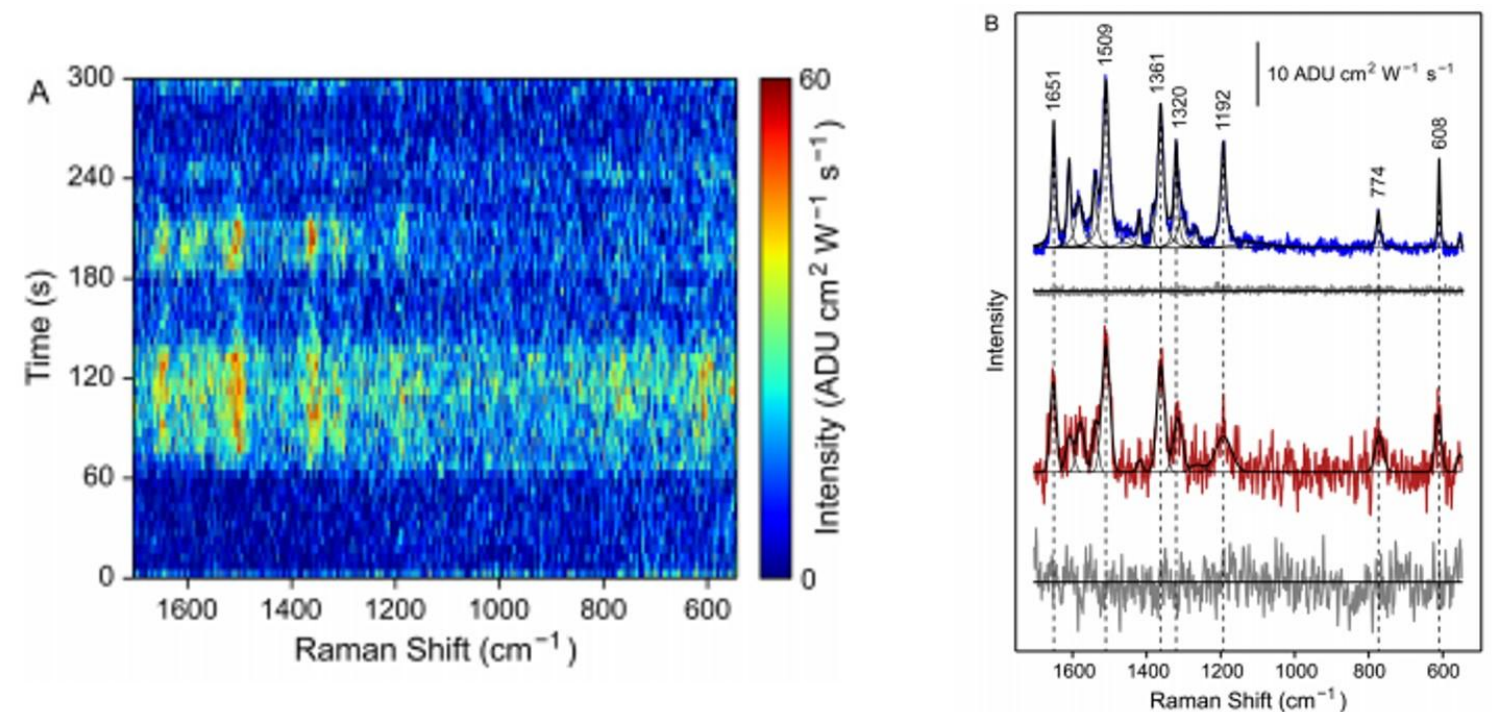

Figure 3. (A) Waterfall plot of sixty $5 \mathrm{~s}$ TER spectra of R6G collected using ps irradiation $\left(\lambda=532 \mathrm{~nm}, \mathrm{P}_{\mathrm{ex}}=0.05 \mathrm{~W} / \mathrm{cm}^{2}\right)$. The initially retracted $\mathrm{Ag}$ tip was brought into tunneling range at $\mathrm{t}=60 \mathrm{~s}$. (B) CW TER engaged (blue) and retracted (gray) spectra ( $\lambda$ $=532 \mathrm{~nm}, \mathrm{P}_{\mathrm{ex}}=1.3 \mathrm{~W} / \mathrm{cm}^{2}, \mathrm{t}_{\mathrm{acq}}=30 \mathrm{~s}$ ) plotted above averages of $12 \mathrm{ps}$ TER engaged spectra with signal/noise ratios above 12 (red) and 12 retracted spectra (gray) from (A). Black lines represent individual and composite peak fits. The included scale bar applies to all plotted spectra. Reprinted with permission from ref 30. Copyright 2014 American Chemical Society.

Significant progress has been made recently toward incorporating nonlinear techniques with TERS. Pulsed laser sources are required for ultrafast TERS, and along these lines Klingsporn et al. demonstrated spontaneous TERS using picosecond excitation and observed the signal to decay over the course of tens of seconds as a result of reactive decay chemistry due to small molecules present in ambient.[18] Along these lines, Pozzi et al. reported the benefits of performing pulsed-excitation TERS in UHV, wherein no irreversible signal loss was observed.[30] Tip-enhancement was also evidenced in stimulated Raman studies. Researchers in the Kawata group obtained images of the CARS signal for a particular adenine Raman band and reported a plasmonic enhancement factor of $\sim 100$ in the presence of a Ag-coated tip.[31] Wickramasinghe et al. demonstrated high sensitivity in stimulated TERS, reporting billion-fold enhancement of an azobenzene thiol band when compared to spontaneous TERS.[32]

While significant advancements have been made in the field, some issues remain unresolved. For example, high peak powers within individual laser pulses cause intense 
electromagnetic fields and likely heat at the tip-sample junction, potentially affecting the stability of molecules in the enhancing region. Unwanted chemical reactions and surface diffusion may result, especially when performed in ambient. As a result, particular molecular systems are expected to be more stable than others under pulsed irradiation. Changes in the fine structure of the probe are also possible in analogy to previous studies on plasmonic colloids and surfaces,[26,33] and preferred probe geometries and compositions may be discerned.

The successful combination of broadband stimulated Raman techniques with TERS will enable investigation of transient behavior in molecular systems with nanoscale spatial resolution. Sub-populations and potentially single molecules may be interrogated, allowing surface sites and adsorption configurations to be differentiated and correlated with transient kinetics. Resulting experiments have the potential to characterize ultrafast processes at the molecular level with unprecedented detail.

\section{Applications}

\subsection{Applications of TERS in Material and Life Sciences.}

A growing body of evidence demonstrates that TERS can be successfully utilized in various research areas ranging from materials and life sciences to conservation art science. For example, it has been shown that TERS is capable of detecting small defects and contaminations in graphene sheets.[34-37] Shaffel et al. found that intensity of the graphene D band $\left(1350 \mathrm{~cm}^{-1}\right)$ significantly increased in the presence of graphene defects, as well as at the edges of graphene sheets.[38] At the same time, in-plane modes, such as $G$ band, were found to be weakly enhanced because of their non-optimal orientation perpendicular to the tip.[39] These studies showed that TERS can be used to probe electronic properties of carbon 2D materials.

Recently, Peica et al. demonstrated that TERS can be utilized to probe structural organization of 3D carbon materials, such as carbon nanotubes.[40] Specifically, using TERS, single-walled carbon nanotubes could be identified within a carbon nanotube bundle. Separately, Yano et al. showed that mechanical properties of carbon nanotubes can be explored by TERS.[41] It has been found that an increase in pressure on the nanotube induced by AFM tip caused a shift in the G mode frequency on around $10 \mathrm{~cm}^{-1}$. These studies revealed that TERS can be utilized to investigate properties of $2 \mathrm{D}$ and $3 \mathrm{D}$ carbon materials at the nanoscale.

Additionally, Van Schrojenstein et al. showed that TERS could be used to monitor photocatalytic conversion of $p$-nitrothiophenol adsorbed on gold nanoplates [42]. This study demonstrated that TERS allows one to overcome averaging effects of standard experiments and is able to study chemical reactions and molecule dynamics at the nanoscale.

Label-free high-resolution examination of chemical composition possible in TERS makes it an attractive technique for the structural characterization of complex biological systems, such as cells, viruses or bacteria. For example, a TERS study of a single tobacco mosaic virus revealed the presence of both coat proteins and RNA components on its surface.[43] Separately, Böhme et al. demonstrated that protein and lipid components can be resolved on the cell surface using 
TERS.[44] In the acquired TER spectra authors also observed vibrational modes originating from oligosaccharides. Using advance spectral processing algorithms Richter et al. showed that using TERS both protein and lipid domains could be differentiated at the nanometer scale.[45]

Macromolecules and protein aggregates, such as DNA, RNA, amyloid and collagen fibrils, were also extensively studied by TERS. For example, the high spatial resolution of TERS allowed researchers to resolve individual nucleobases on both RNA and DNA strands.[46,47] It was demonstrated that all four nucleobases could be identified on both RNA and DNA strands. $[48,49]$ Recently, Lipiec et al. reported that, using TERS, molecular structure of terminal groups at DNA fragments can be probed, which consequently allowed the authors to identify double strand breaks of DNA.[50] Based on the acquired TER spectra authors proposed that UV radiation likely causes $\mathrm{C}=\mathrm{O}$ band cleavage.

Amyloid fibrils are $\beta$-sheet rich protein aggregates that are strongly associated with various neurodegenerative diseases.[51] TERS studies of insulin fibrils revealed that their surface is heterogeneous from the viewpoint of both protein secondary structure and amino acid composition.[52,53] It has been shown that only around a third of insulin fibril surface was composed of $\beta$-sheet, which may template aggregation of misfolded protein, while the rest of it was dominated by mixtures of $\alpha$-helix and unordered protein. This discovery indicated that amyloid fibrils have different inside (cross- $\beta$-sheet core) and outside (surface) structural organization. Kurouski et al. also demonstrated that $\beta$-sheet clusters and areas dominated by $\alpha$ helix/ unordered protein secondary structures have different amino acid compositions. It has been found that aromatic amino acids, such as tyrosine and phenylalanine, as well as cysteine are much more frequently present on $\beta$-sheet clusters than on the areas dominated by $\alpha$-helix/ unordered protein. At the same time, proline, which is known to disrupt $\beta$-sheet integrity, is much more abundant in $\alpha$-helix/ unordered protein clusters. TERS was also utilized to probe surface organization of insulin fibril polymorphs with different topologies: flat, tape like and twisted.[54] It has been found that surfaces of these polymorphs have distinctively different amino acid composition and protein secondary structures. Also, for the first time the surface of filaments, precursors of fibril, was investigated. Comparison of amino acid propensities and protein secondary structures on the surface of the filaments with mature fibrils elucidated the mechanism of filament propagation into mature insulin fibrils.

These studies also revealed the challenges faced by TERS for the investigation of structural organization of complicated macroscopic biosystems. First, many biological molecules, such as amino acids and lipids, often have the same chemical groups, which consequently will give the same vibrational bands in Raman spectra. Therefore, it is often difficult to achieve unambiguous assignment of the vibrational bands and consequently identify these molecules.[55,56] Secondly, TER spectra acquired form protein molecules often lack amide bands, which limits their utilization for determining protein secondary structure. Kurouski et al. has recently proposed that this can be due to a distancing of the peptide bond from the rough metal surface of STM or AFM tip by amino acid side chains. [57] However, it is likely that other factors, which are yet unknown, can contribute to the amide bonds suppression.[56] Finally, the short lifetime of SPM probes and 
their possible contamination during spectral acquisition limits TERS imaging of large areas of biological objects, such as cells.

\subsection{Applications of TERS in Art Conservation Science.}

Confirmatory, non-destructive and non-invasive identification of colorants in situ is highly important for the understanding of historical context and for the long-term preservation of cultural heritage objects.[58-60] It was recently demonstrated that TERS can identify indigo dye and iron gall ink in situ on Kinwashi paper.[61] In this proof-of-concept work, Ag-coated AFM tip was approached at the surface of the paper and illuminated it with $532 \mathrm{~nm}$ laser radiation. TER spectra were directly acquired from the paper using low laser powers $(\sim 0.2 \mathrm{~mW})$ and fast acquisition times (3-10 sec) avoiding any damage or photo-degradation to the dyed paper. Authors demonstrated that TER spectra collected from Kinwashi paper dyed in $0.5 \mathrm{M}$ indigo solution exhibit vibrational bands originating from indigo, cellulose and its monomer glucose. This study revealed that, using TERS, organic dyes can be non-destructively and non-invasively identified directly on artwork. It also showed that TERS was able to resolve chemical composition of the paper down to its monomers.

Authors also demonstrated that TERS can be utilized for identification of iron gall ink on historical documents. For example, a fragment of the latter dated to the $19^{\text {th }}$ century with a hand written text was investigated by positioning a Ag-coated AFM tip on a single fiber of paper near the circumference of one of the cracks, as shown in Figure 4D (red arrows). The acquired TER spectra exhibited vibrational modes corresponding to gallic acid, the main component of iron gall ink, and glucose, a cellulose monomer. These results indicated that TERS allowed for the identification of individual components in the complex chemical mixture such as iron gall ink, which could not be achieved using normal Raman spectroscopy even with higher powers and longer acquisition times. This work demonstrated the great potential of TERS as an additional spectroscopic tool for minimally invasive compositional characterization of artworks in situ and opened exciting new possibilities for cultural heritage research. 

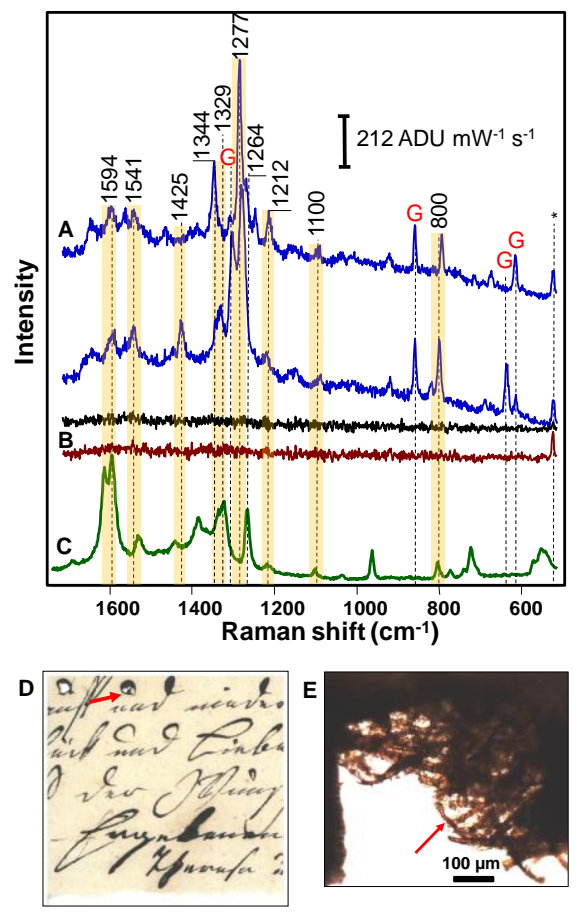

Figure 4. TER spectra of (A) K. paper dyed with iron gall ink engaged (red) and retracted (black). The asterisk (*) indicates the peak at $521 \mathrm{~cm}^{-1}$ that results from silicon on the AFM tip. TER spectra acquired using $\lambda_{\mathrm{ex}}=532 \mathrm{~nm} ; \mathrm{t}_{\mathrm{acq}}=4 \mathrm{~s} ; \mathrm{P}_{\mathrm{ex}} \approx 0.25 \mathrm{~mW}$. Normal Raman spectra of (B) gallic acid powder and (C) iron gall ink in solution. Raman spectra were acquired using $\lambda_{\mathrm{ex}}=785$ $\mathrm{nm} ; \mathrm{t}_{\mathrm{acq}}=10 \mathrm{~s}$ and $60 \mathrm{~s}$ respectively; and $\mathrm{P}_{\mathrm{ex}} \approx 1 \mathrm{~mW}$. Peaks corresponding to gallic acid in the TER spectra are marked with $\diamond$. Peaks corresponding to the vibrational modes of both cellulose and glucose and just glucose are marked with " $\mathrm{C} / \mathrm{G}$ " and " $\mathrm{G}$ " respectively. Fragment of 19th century letter (D) and a micrograph of the paper crack (E). Reprinted with permission from ref 51. Copyright 2014, American Chemical Society.

\subsection{Electrochemical Scanning Probe Microscopy and TERS}

Chemical and physical processes that take place at solid/liquid interfaces have been intensively investigated in recent years.[62,63] These studies allowed researchers to understand fundamental mechanisms of electron and ion transfers at the solid/liquid interfaces and consequently were the basis for different batteries, semiconductors, fuel cells, and solar cells.[64] Cyclic voltammetry in general and scanning electrochemical microscopy (SECM) and scanning electrochemical cell microscopy (SECCM) in particular, are commonly used to study electrochemical reactions at solid-liquid interfaces [65-67]. Precise measurements of the current and voltage allow one to acquire valuable information about chemical and physical processes at the interfaces, such as diffusion, adsorption, electron and ion transfers. Applications of other techniques, such as quartz crystal microbalance (QCM)[68], infrared, Raman and surface enhanced Raman spectroscopies[69,70] have drastically expanded our understanding about electrochemical processes at solid/liquid interfaces. The invention of SPM signficantly impacted studies of solid/liquid interface processes because of its ability to acquire information about the surface structure with atomic resolution in real time and real space. In particular, it was found that adsorption-desorption reactions of hydrogen is strongly dependent on the crystallographic orientation of platinum.[71] Development of electrochemical SPM (ECSPM) by coupling STM with electrochemistry brought substantial progress in surface and interface research.[72-74] In 
this setup, the bias potential of the STM tip and the sample can be separately controlled using a bipotentiostat. This allowed researchers to study surface reactions at the nanoscale and manipulate surface objects, as well as monitor oxidation-reduction conversion of the single molecule[66,75].
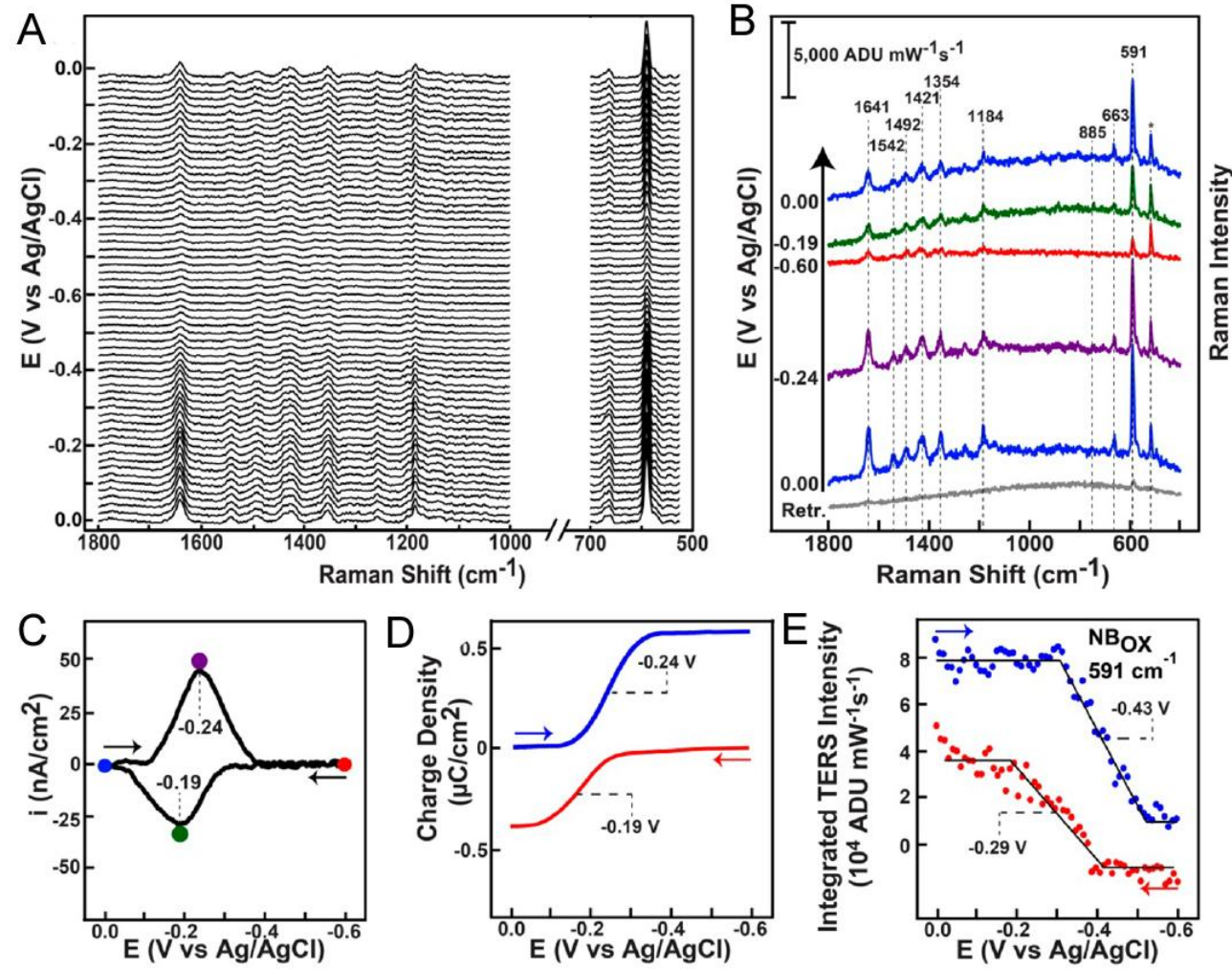

Figure 5. Stack plot (a) and individual (b) EC-TER spectra of NB acquired at different potentials during cyclic voltammetry (CV) showing reversible reduction and oxidation of NB. $\mathrm{P}=110 \mu \mathrm{W}, \mathrm{t}=1 \mathrm{~s}, \lambda=633 \mathrm{~nm}$, scan rate $=10 \mathrm{mV} / \mathrm{s}$. The asterisk denotes the Si Raman band from the AFM tip. (c) Background subtracted CV of NB on ITO acquired concurrently with the EC-TER spectra in (a). E0' $=-0.215 \mathrm{~V}$. (d) Charge acquired by integrating the $\mathrm{CV}$ in (c). The charge is directly proportional to the number of molecules reduced during the CV. The reverse sweep is offset by $0.6 \mu \mathrm{C} / \mathrm{cm} 2$ for clarity. (e) Potential dependence of the integrated TERS intensity of the $591 \mathrm{~cm}^{-1}$ band of NBOX. The black lines are meant to highlight the dependence as a guide for the reader. The intensity-potential profiles strongly resemble the mirror image of the charge shown in (d). All TERS intensities were normalized to the initial value at $0.0 \mathrm{~V} \mathrm{vs} \mathrm{Ag} / \mathrm{AgCl}$. The reverse sweep is offset for clarity. TERS spectra in (a) and TERS voltammograms in (e) are the average of four experiments. Reprinted with permission from ref 72 . Copyright 2015 , American Chemical Society.

The Van Duyne group has recently demonstrated the first AFM electrochemical TERS (ECTERS) [76]. Kurouski et al. investigated nanoscale redox behavior of Nile Blue (NB) and compared these results with conventional cyclic voltammetry $(\mathrm{CV})$. NB undergoes two electron, one proton reduction at negative potentials $(\sim-0.5 \mathrm{~V}$ vs $\mathrm{Ag} / \mathrm{AgCl})$ at $\mathrm{pH}$ above 6 . While the oxidized form of $\mathrm{NB}\left(\mathrm{NB}_{\mathrm{O}}\right)$ absorbs light at $\sim 630 \mathrm{~nm}$, the reduced one $\left(\mathrm{NB}_{\mathrm{R}}\right)$ lacks absorption in this spectral region. Instead, it exhibits several absorption bands in the near-UV (261, 362 and $405 \mathrm{~nm}$ ). In the EC-TERS experiment, NB was adsorbed onto indium tin oxide (ITO) film, which was used as the working electrode (WE). $\mathrm{Pt}$ and $\mathrm{Ag} / \mathrm{AgCl}$ were used as the counter and reference electrodes respectively. After the Au-coated AFM tip was positioned on the WE, 
changes in acquired TER spectra were monitored as the potential was swept from 0.0 to $-0.6 \mathrm{~V}$ vs $\mathrm{Ag} / \mathrm{AgCl}$ and returned back to $0.0 \mathrm{~V} v s \mathrm{Ag} / \mathrm{AgCl}$ (Figure 5). Kurouski et al. found that overall intensities of the spectra decreased with a decrease of the potential, in agreement with the change in electronic state, from $\mathrm{NB}_{\mathrm{O}}$ to $\mathrm{NB}_{\mathrm{R}}$. As the potential was swept from $-0.6 \mathrm{~V}$ back to $0.0 \mathrm{~V}, \sim 75 \%$ of the initial spectral intensity returned, demonstrating reversibility of the monitored redox reaction of NB under the AFM.

Recently, the Ren group reported that STM EC-TERS could be utilized to monitor arrangements of $4^{\prime}$-(pyridin-4-yl)biphenyl-4-yl) methanethiol (4-PBT) on $\mathrm{Au}(111)$ as a result of its protonation and deprotonation [77]. The authors pointed out that such information about potential-dependent re-configuration of 4-PBT could not be obtained from EC-SERS experiments. This work demonstrated that insulation of STM tips, necessary for reducing Faradaic current from tip shaft, could be successfully achieved. Zeng et al. proposed to embed freshly etched $\mathrm{Au}$ or $\mathrm{Ag}$ wire into polyethylene glue, which allowed the tip apex to remain plasmonically active [77].

These findings demonstrate that TERS can be successfully used to monitor electrochemical processes at the nanoscale. They show that, using TERS, one can understand how the number of molecular binding sites changes the electrochemical properties of the molecule, including its formal potentials and the electron transfer kinetics. One can expect that these findings will help to develop our understanding of plasmon-driven electron transfer as well as to improve the performance of solar cells and batteries.

\section{Concluding Remarks}

With the aid of TERS, the unambiguous and quantitative interrogation of individual molecules can be explored in detail. Simultaneous detection of SPM topography and vibrational signatures makes TERS a powerful tool for studying light-matter interaction at the single-molecule level. TERS can be performed in a variety of environments (UHV, controlled atmosphere, liquid) and at variable temperatures. The capability of combining pulsed laser excitation with TERS gives the opportunity of performing measurements with simultaneous high spatial and temporal resolution.

The recent applications of TERS has been demonstrated in various research areas ranging from electrochemistry to art conservation science. It shows that TERS can be used to investigate structural organization of biological specimens, such as cell membranes and amyloid fibrils. Using this powerful analytical technique, amino acid composition and protein secondary structure on the surface of these physiologically active species could be unraveled. Moreover, TERS can be used to unravel physical properties and defects in polymeric materials. The review also shows how TERS can be utilized to monitor electrochemical and photocatalytic reactions. Sub-nanometer spatial resolution and single-molecule sensitivity of TERS makes it highly desirable for probing electrochemical and electrocatalytic reactions at different surface sites, such as lattices, edges and vacancies. One can expect that these findings will transform our understanding about catalysis, electron transfer and energy storage. Therefore, TERS has the 
potential to have great scientific impact through understanding site-specific chemical reactivity at surfaces.

\section{Acknowledgments}

E.A.P. and R.P.V.D. acknowledge support from the National Science Foundation Center for Chemical Innovation dedicated to Chemistry at the Space-Time Limit (CaSTL) Grant CHE1414466. D. K. and R.P.V.D were supported by the Air Force Office of Scientific Research MURI (FA9550-14-1-0003). N.C., N.J., T.S., M.C.H. and R.P.V.D. acknowledge funding from the Department of Energy Office of Basic Energy Sciences (SISGR Grant DE-FG0209ER16109). E.A.P., M.C.H., and R.P.V.D. also acknowledge support from the National Science Foundation Materials Research Science and Engineering Center (DMR-1121262). E.A.P. was additionally supported by the National Science Foundation Graduate Research Fellowship under Grant DGE-1324585.

\section{References:}

[1] S.W. Hell, J. Wichmann, Opt Lett 19 (1994) 780.

[2] M.J. Rust, M. Bates, X.W. Zhuang, Nat Methods 3 (2006) 793.

[3] E. Betzig, G.H. Patterson, R. Sougrat, O.W. Lindwasser, S. Olenych, J.S. Bonifacino, M.W. Davidson, J. Lippincott-Schwartz, H.F. Hess, Science 313 (2006) 1642.

[4] B.C. Stipe, M.A. Rezaei, W. Ho, Science 280 (1998) 1732.

[5] F. Lu, M.Z. Jin, M.A. Belkin, Nat Photonics 8 (2014) 307.

[6] R.M. Stockle, Y.D. Suh, V. Deckert, R. Zenobi, Chem Phys Lett 318 (2000) 131.

[7] B. Pettinger, G. Picardi, R. Schuster, G. Ertl, Electrochemistry 68 (2000) 942.

[8] N. Hayazawa, Y. Inouye, Z. Sekkat, S. Kawata, Opt Commun 183 (2000) 333.

[9] M.S. Anderson, Appl Phys Lett 76 (2000) 3130.

[10] C.C. Neacsu, J. Dreyer, N. Behr, M.B. Raschke, Phys Rev B 73 (2006) 193406.

[11] J. Steidtner, B. Pettinger, Phys Rev Lett 100 (2008) 236101.

[12] M.D. Sonntag, J.M. Klingsporn, L.K. Garibay, J.M. Roberts, J.A. Dieringer, T. Seideman, K.A. Scheidt, L. Jensen, G.C. Schatz, R.P. Van Duyne, J Phys Chem C 116 (2012) 478.

[13] T. Deckert-Gaudig, E. Kammer, V. Deckert, J Biophotonics 5 (2012) 215.

[14] C. Chen, N. Hayazawa, S. Kawata, Nat Commun 5 (2014) 3312.

[15] R. Zhang, Y. Zhang, Z.C. Dong, S. Jiang, C. Zhang, L.G. Chen, L. Zhang, Y. Liao, J. Aizpurua, Y. Luo, J.L. Yang, J.G. Hou, Nature 498 (2013) 82.

[16] N. Jiang, E.T. Foley, J.M. Klingsporn, M.D. Sonntag, N.A. Valley, J.A. Dieringer, T. Seideman, G.C. Schatz, M.C. Hersam, R.P. Van Duyne, Nano letters 12 (2012) 5061.

[17] N.H. Chiang, N. Jiang, D.V. Chulhai, E.A. Pozzi, M.C. Hersam, L. Jensen, T. Seideman, R.P. Van Duyne, Nano letters 15 (2015) 4114.

[18] J.M. Klingsporn, N. Jiang, E.A. Pozzi, M.D. Sonntag, D. Chulhai, T. Seideman, L. Jensen, M.C. Hersam, R.P. Van Duyne, Journal of the American Chemical Society 136 (2014) 3881.

[19] Z. Liu, S.Y. Ding, Z.B. Chen, X. Wang, J.H. Tian, J.R. Anema, X.S. Zhou, D.Y. Wu, B.W. Mao, X. Xu, B. Ren, Z.Q. Tian, Nat Commun 2 (2011) 305.

[20] E.M. van Schrojenstein Lantman, T. Deckert-Gaudig, A.J.G. Mank, V. Deckert, B.M. Weckhuysen, Nat Nanotechnol 7 (2012) 583. 
[21] P. Kukura, D.W. McCamant, R.A. Mathies, Annu Rev Phys Chem 58 (2007) 461.

[22] J.X. Cheng, X.S. Xie, Journal of Physical Chemistry B 108 (2004) 827.

[23] P. Kukura, D.W. McCamant, S. Yoon, D.B. Wandschneider, R.A. Mathies, Science 310 (2005) 1006.

[24] C. Fang, R.R. Frontiera, R. Tran, R.A. Mathies, Nature 462 (2009) 200.

[25] J.V. Lockard, A. Butler Ricks, D.T. Co, M.R. Wasielewski, The Journal of Physical Chemistry Letters 1 (2009) 215.

[26] R.R. Frontiera, A.I. Henry, N.L. Gruenke, R.P. Van Duyne, Journal of Physical Chemistry Letters 2 (2011) 1199.

[27] R.R. Frontiera, N.L. Gruenke, R.P. Van Duyne, Nano letters 12 (2012) 5989.

[28] S. Yampolsky, D.A. Fishman, S. Dey, E. Hulkko, M. Banik, E.O. Potma, V.A. Apkarian, Nat Photonics 8 (2014) 650.

[29] Y. Zhang, Y.R. Zhen, O. Neumann, J.K. Day, P. Nordlander, N.J. Halas, Nat Commun 5 (2014) 4424.

[30] E.A. Pozzi, M.D. Sonntag, N. Jiang, N. Chiang, T. Seideman, M.C. Hersam, R.P. Van Duyne, Journal of Physical Chemistry Letters 5 (2014) 2657.

[31] T. Ichimura, N. Hayazawa, M. Hashimoto, Y. Inouye, S. Kawata, Phys Rev Lett 92 (2004).

[32] H.K. Wickramasinghe, M. Chaigneau, R. Yasukuni, G. Picardi, R. Ossikovski, ACS nano 8 (2014) 3421.

[33] J.C. Hulteen, M.A. Young, R.P. Van Duyne, Langmuir 22 (2006) 10354.

[34] A.C. Ferrari, J.C. Meyer, V. Scardaci, C. Casiraghi, M. Lazzeri, F. Mauri, S. Piscanec, D. Jiang, K.S. Novoselov, S. Roth, A.K. Geim, Phys Rev Lett 97 (2006) 187401.

[35] R. Zhao, Y. Zhang, T. Gao, Y. Gao, N. Liu, L. Fu, Z. Liu, Nano Res. 4 (2011) 712.

[36] Y. Zhang, T. Gao, Y. Gao, S. Xie, Q. Ji, K. Yan, H. Peng, Z. Liu, ACS nano 5 (2011) 4014.

[37] M. Tommasini, C. Castiglioni, G. Zerbi, Physical chemistry chemical physics 11 (2009) 10185.

[38] F. Schaffel, M. Wilson, A. Bachmatiuk, M.H. Rummeli, U. Queitsch, B. Rellinghaus, G.A. Briggs, J.H. Warner, ACS nano 5 (2011) 1975.

[39] K.F. Domke, B. Pettinger, J. Raman Spectrosc. 40 (2009) 1427.

[40] N. Peica, C. Thomsen, J. Maultzsch, Nanoscale research letters 6 (2011) 174.

[41] T. Yano, P. Verma, Y. Saito, T. Ichimura, S. Kawata, Nat. Photon. 2009 (2009) 473.

[42] E.M. van Schrojenstein Lantman, T. Deckert-Gaudig, A.J. Mank, V. Deckert, B.M. Weckhuysen, Nature nanotechnology 7 (2012) 583.

[43] D. Cialla, T. Deckert-Gaudig, C. Budich, M. Laue, R. Moller, D. Naumann, V. Deckert, J. Popp, J. Raman. Spectr. 40 (2009) 240.

[44] R. Bohme, D. Cialla, M. Richter, P. Rosch, J. Popp, V. Deckert, J Biophotonics 3 (2011) 455.

[45] M. Richter, M. Hedegaard, T. Deckert-Gaudig, P. Lampen, V. Deckert, Small 7 (2011) 209.

[46] R. Treffer, R. Bohme, T. Deckert-Gaudig, K. Lau, S. Tiede, X. Lin, V. Deckert, Biochemical Society transactions 40 (2012) 609.

[47] R. Treffer, V. Deckert, Current opinion in biotechnology 21 (2010) 4.

[48] R. Treffer, X. Lin, E. Bailo, T. Deckert-Gaudig, V. Deckert, Beilstein journal of nanotechnology 2 (2011) 628.

[49] A. Rasmussen, V. Deckert, J. Raman Spectrosc. 37 (2006) 311.

[50] E. Lipiec, R. Sekine, J. Bielecki, W.M. Kwiatek, B.R. Wood, Angewandte Chemie 53 (2014) 169.

[51] T.P. Knowles, M. Vendruscolo, C.M. Dobson, Nature reviews. Molecular cell biology 15 (2014) 384.

[52] T. Deckert-Gaudig, E. Kammer, V. Deckert, J Biophotonics 5 (2012) 215.

[53] D. Kurouski, T. Deckert-Gaudig, V. Deckert, I.K. Lednev, Journal of the American Chemical Society 134 (2012) 13323. 
[54] D. Kurouski, T. Deckert-Gaudig, V. Deckert, I.K. Lednev, Biophysical journal 106 (2014) 263.

[55] C. Blum, T. Schmidt, L. Opilik, S. Weidmann, S.R. Fagerer, R. Zenobi, J. Raman Spectr. 43 (2012) 1895.

[56] C. Blum, L. Opilik, J.M. Atkin, K. Braun, S.B. Kammer, V. Kravtsov, N. Kumar, S. Lemeshko, J.-F. Li, K. Luszcz, T. Maleki, A.J. Meixner, S. Minne, M.B. Raschke, B. Ren, J. Rogalski, D. Roy, B. Stephanidis, X. Wang, D. Zhang, J.-H. Zhong, R. Zenobi, Journal of Raman Spectroscopy 45 (2014) 22.

[57] D. Kurouski, T. Postiglione, T. Deckert-Gaudig, V. Deckert, I.K. Lednev, The Analyst 138 (2013) 1665.

[58] C.L. Brosseau, F. Casadio, R.P. Van Duyne, Journal of Raman Spectroscopy 42 (2011) 1305.

[59] C.L. Brosseau, A. Gambardella, F. Casadio, C.M. Grzywacz, J. Wouters, R.P. Van Duyne, Anal. Chem. (Washington, DC, U. S.) 81 (2009) 3056.

[60] F. Casadio, M. Leona, R. Lombardi John, R.P. Van Duyne, , Accounts of Chemical Research 43 (2010) 782.

[61] D. Kurouski, S. Zaleski, F. Casadio, R.P. Van Duyne, N.C. Shah, J. Am. Chem. Soc. (2014).

[62] D. Wang, L.-J. Wan, J. Phys. Chem. C 111 (2007) 16109.

[63] S. Yoshimoto, K. Itaya, Annual review of analytical chemistry (Palo Alto, Calif 6213.

[64] A.J. Bard, H.D. Abruna, C.E. Chidsey, L.R. Faulkner, S.W. Feldberg, K. Itaya, M. Majda, O. Melroy, R.W. Murray, J. Phys. Chem. 40 (1993) 1162.

[65] T. Sun, Y. Yu, B.J. Zacher, M.V. Mirkin, Angewandte Chemie (International ed 53 (2014) 14120.

[66] M. Wilms, M. Kruft, G. Bermes, K. Wandelt, The Review of scientific instruments 70 (1999) 3641.

[67] M.E. Snowden, A.G. Guell, S.C. Lai, K. McKelvey, N. Ebejer, M.A. O'Connell, A.W. Colburn, P.R. Unwin, Anal Chem 84 (2012) 2483.

[68] R. Schuumacher, Angewandte Chemie 29 (1990) 329.

[69] T. Iwasita, F.C. Nart, Prog. Surf. Sci. 55 (1997) 271.

[70] P.L. Stiles, J.A. Dieringer, N.C. Shah, R.P. Van Duyne, Annual review of analytical chemistry (Palo Alto, Calif 1 (2008) 601.

[71] J. Clavilier, R. Faure, G. Guinet, R. Durand, J. Electroanal. Chem. 107 (1980) 205.

[72] R. Sonnenfeld, P.K. Hansma, Science 232 (1986) 211.

[73] K. Itaya, S. Sugawara, Chem. Lett. 16 (1987) 1927.

[74] H.Y. Liu, F.R.F. Fan, C.W. Lin, A.J. Bard, J. Am. Chem. Soc. 108 (1986) 3838.

[75] K. Suto, S. Yoshimoto, K. Itaya, Langmuir 22 (2006) 10766.

[76] D. Kurouski, M. Mattei, R.P. Van Duyne, Nano letters 15 (2015) 7956.

[77] Z.C. Zeng, S.C. Huang, D.Y. Wu, L.Y. Meng, M.H. Li, T.X. Huang, J.H. Zhong, X. Wang, Z.L. Yang, B. Ren, Journal of the American Chemical Society 137 (2015) 11928. 


\section{Tip Enhanced Raman Spectroscopy}

\section{Concepts:} Spatial Resolution Temporal Resolution

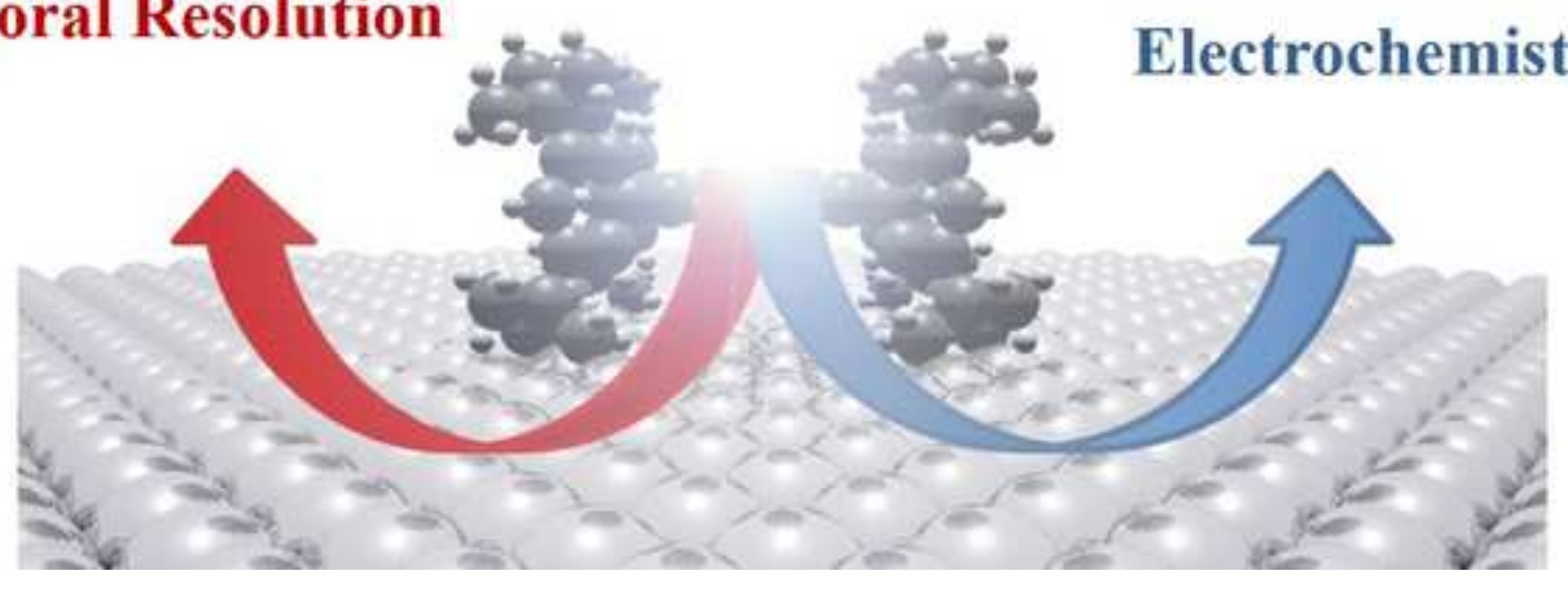

Applications: Material and Life Science Art Conservation Electrochemistry 